\section{Wortgenerierung bei Bilingualen - eine fMRT-Studie mit Implikationen für Sprach- und Gedächtnisprozesse}

Zusammenfassung: Die Diskussion zur Frage der zerebralen Repräsentation von Sprachfunktionen bei mehrsprachigen Personen blickt mittlerweile auf einen Zeitraum von mehr als 100 Jahren zurück. Traditionsgemäß wird die Sprache in der Hirnforschung als eine der am stärksten lateralisierten Funktionen angesehen. Während die Repräsentation der zuerst erworbenen Sprache in der Regel links-hemisphärisch lateralisiert ist, sprechen einige Studien an Hirngeschädigten, aber auch klinischexperimentelle Untersuchungen der rechten Hirnhälfte eine spezielle Bedeutung hinsichtlich der Realisierung der Zweitsprache zu. Mit der Entwicklung der modernen Verfahren zur funktionellen Bildgebung eröffnet sich nun die Perspektive, auch Normalpersonen hinsichtlich grundlegender Fragen zur zerebralen Repräsentation bei Bilingualität zu untersuchen. Vor diesem Hintergrund wurde eine Gruppe bilingualer Normalpersonen anhand eines Wortflüssigkeitsparadigmas mit Hilfe der funktionellen Magnetresonanztomographie (fMRT) in ihrer Erst- und Zweitsprache evaluiert. Während sowohl in der Erstals auch in der Zweitsprache eine konsistente Aktivierung des linken präfrontalen Kortex zur Darstellung kam, zeigte sich in der Zweitsprache in der Regel eine zusätzliche Aktivierung in der rechten Präfrontalregion. Die gewonnenen Ergebnisse werden vor dem Hintergrund gegenwärtiger Konzepte der Informationsverarbeitung, insbesondere der Wechselwirkung zwischen Sprach- und Gedächtnisprozessen interpretiert und diskutiert.

Word Generation in Bilinguals - fMRI Study with Implications for Language and Memory Processes: For over a century the cerebral representation of language functions is a matter of debate. In Neuroscience language is regarded as one of the most lateralized cognitive functions. Thus, while the language which is acquired first in most cases is processed by the left hemisphere some studies in brain damaged but also experimental investigations propose a pivotal role of the right hemisphere in second language processing. By the advent of modern neuroimaging it is now possible to study language lateralization and bilinguality also in healthy subjects. We studied first and second language abilities in a group of bilingual, healthy individuals by means of functional magnetic resonance imaging (fMRI) with a word-fluency paradigm. While we found a predominantly left prefrontal activity during both first and also second language processing an additional right prefrontal activa-

Fortschr Neurol Psychiat 2001; 69: $42-50$ (c) Georg Thieme Verlag Stuttgart · New York ISSN 0720-4299
P. Calabrese ${ }^{1}$, H. Neufeld ${ }^{1}$, A. Falk ${ }^{2}$, H. J. Markowitsch ${ }^{3}$, C. Müller ${ }^{2}$, L. Heuser ${ }^{2}$, W. Gehlen ${ }^{1}$, H. F. Durwen ${ }^{4}$

${ }^{1}$ Neurologische Universitätsklinik, Knappschaftskrankenhaus Bochum-Langendreer, Klinikum der Ruhr-Universität Bochum (Direktor: Prof. Dr. W. Gehlen)

2 Institut für Radiologie und Nuklearmedizin, Knappschaftskrankenhaus Bochum-Langendreer, Klinikum der Ruhr-Universität Bochum (Direktor: Prof. Dr. L. Heuser)

${ }^{3}$ Lehrstuhl für Physiologische Psychologie, Universität Bielefeld (Direktor: Prof. Dr. H. J. Markowitsch)

${ }^{4}$ Klinik für Akut-Neuro-Geriatrie, St. Martinus-Krankenhaus, Düsseldorf (Ärztl. Leiter: PD. Dr. H. F. Durwen)

tion was registered during the use of second language. Our findings are discussed on the basis of an interaction between language and memory processes.

\section{Einleitung}

Die Erforschung des Phänomens Bilingualität ${ }^{1}$ hat eine lange Tradition. Die ersten Überlegungen zur Frage der neuronalen und funktionellen Repräsentation der Sprachen bei Polyglotten reichen bis zum Ende des vorigen Jahrhunderts zurück. Kurze Zeit nach den Entdeckungen Brocas [1] und Wernickes [2] zur Lokalisation der wichtigsten Sprachzentren publizierte Jean-Albert Pitres [3] seine Beobachtungen über die unterschiedlichen Beeinträchtigungs- und Restitutionsmuster der Aphasien bei mehrsprachigen Personen. Die Variabilität der Symptomatik und Erholungsdynamik wies auf mögliche Unterschiede in der Organisation und Repräsentation der verschiedenen Sprachen hin. Die Annahme Pitres, dass die vertrautere bzw. vor der Hirnschädigung mehr in Anspruch genommene Sprache weniger beeinträchtigt sei und sich schneller erhole, impliziert bereits den Einfluss mehrerer unterschiedlicher Variablen, die aber nicht nur eine mögliche Restitutionssituation, sondern auch die grundlegende Strukturierung und funktionelle Organisation der beiden Sprachen determinieren können. Die Frage nach der funktionalen und anatomischen Repräsentation der Sprachen bei Bilingualen dürfte, wie es die weitere Forschung normaler und gestörter Sprachprozesse gezeigt hat, nicht ohne Einbeziehung paralinguistischer Faktoren sinnvoll beantwortet werden. Der am meisten kontrovers diskutierte Gesichtspunkt betraf die Lateralisation der Zweitsprache. Während zahlreiche Autoren wiederholt auf eine größere Involvierung der rechten Hemisphäre oder gar auf eine rechtshemisphärische Dominanz bei der Zweitsprache hinwiesen [4], fanden sich weder in den seit den 60 -er Jahren intensiv durchgeführten tachistoskopischen

\footnotetext{
${ }^{1}$ Um begriffliche Unstimmigkeiten zwischen verschiedenen Termini, wie Bi-, Multilingual oder Polyglott zu vermeiden, wird hier und im Folgenden entsprechend einer, sich seit langem in der Fachliteratur zum Thema Bilingualität etablierten Tradition unter diesem Begriff im Allgemeinen das weitgehend flüssige Beherrschen von mehr als einer Sprache verstanden (vgl. auch [11]). Von dieser Grundfähigkeit ausgegangen werden in der Regel unterschiedliche Formen der Bilingualität unterschieden, die im Einzelnen einzuführen sind (z. B. Früh- oder Spätbilingualismus in Abhängigkeit vom Alter des Zweitoder Mehrspracherwerbs).
} 
und dichotischen Darbietungsexperimenten wirklich konsistente Bestätigungen dieser Annahme, noch haben Evidenzen aus klinischen Beobachtungen bei gekreuzten Aphasien oder bei Untersuchungen im Rahmen von sogenannten WADATestungen entsprechend eindeutige Bestätigungen erbracht [4-8]. Eine Analyse diesbezüglicher kontroverser Ergebnisse führte Paradis [8,9] zu der Schlussfolgerung, dass bei ansonsten gesicherter Repräsentation in homologen Spracharealen für beide Sprachen - im Standardfall links - auf die Beteiligung der rechten Hemisphäre in unterschiedlichem Grade und in Abhängigkeit von verschiedenen Faktoren zurückgegriffen werden kann. Diese Faktoren betreffen sowohl die Aspekte des Spracherwerbs, wie z.B. das jeweilige Alter und die Umstände, unter denen die Zweitsprache erworben wurde, aber auch die aktuelle Sprachkompetenz, den kognitiven Stil und die Bedingungen der jeweiligen sprachlichen Aufgabe in der Untersuchungssituation. Hierzu gehört u.a. auch die Realisation von Kompensationsstrategien in Bezug auf sprachpragmatische Aspekte. Ferner wurde zum einen auf die methodischen Limitierungen der bisherigen diagnostischen und experimentellen Ansätze hingewiesen [10], zum anderen wurde im Hinblick auf die nicht unerhebliche individuelle Variabilität die Notwendigkeit der Einzelfallanalyse hinsichtlich der verschiedenen Einflussfaktoren betont [11].

Vor diesem Hintergrund eröffnet die Entwicklung moderner funktionell-bildgebender Verfahren auch in der Bilingualitätsforschung eine qualitativ neue Dimension für die weitere Vertiefung derartiger Fragestellungen. Die neuartigen Möglichkeiten der In-vivo-Registrierung neuronaler Aktivitätsmuster normal ablaufender kognitiver Prozesse erlauben es, die bislang erarbeiteten Vorstellungen zur neuronalen Repräsentation ggf. zu modifizieren und die gewonnenen Erkenntnisse zur Verifizierung neuer theoretischer Modelle über den Aufbau kognitiver Systeme heranzuziehen. So zeichnen sich auch in der Bilingualitätsforschung Tendenzen ab, verschiedene Variablen aufzudecken, die die neuronale Repräsentation der Zweitsprache determinieren [12-14].

Unter Berücksichtigung dieser Entwicklungen und im Hinblick auf den in der vorliegenden Arbeit verfolgten experimentellen Ansatz erscheint die Frage nach dem Einfluss mnestischer Prozesse von nicht unerheblicher Bedeutung. Die moderne Gedächtnisforschung geht von der Existenz einer Reihe verschiedener, z.T. unterschiedlich lokalisierter expliziter und impliziter Gedächtnissysteme aus, die sowohl bei dem Erwerb als auch bei der Realisierung der Zweitsprache unterschiedliche Beteiligungen aufweisen und sich somit in verschiedenen neuronalen Aktivierungsmustern widerspiegeln können. Wir präsentieren hier die Ergebnisse einer fMRT-Aktivierungsstudie bei einer Gruppe Bilingualer unter Anwendung eines Wortflüssigkeitsparadigmas, das sowohl als Sprachproduktions- wie auch als Abrufaufgabe des semantischen Gedächtnisses verstanden werden kann.

\section{Probanden und Methode}

\section{Probanden}

Insgesamt wurde eine Gruppe von vier männlichen und drei weiblichen gesunden Probanden untersucht. Das Probandenkollektiv setzte sich aus russischen Gastwissenschaftlern (RG, Tab.1), die bis zum Zeitpunkt der Untersuchung im Durch- schnitt ca. 3-4 Jahre in Deutschland verbracht haben sowie russlanddeutschen Aussiedlern (RA) mit etwa der gleichen Aufenthaltsdauer, bis auf einen Probanden, der seit der Einreise im Alter von 10 Jahren bisher insgesamt 11 Jahre in Deutschland lebte, zusammen. Das Alter der Teilnehmer lag zwischen 19 und 32 Jahren (Median =27), die Dauer des aktiven Zweitsprachgebrauchs betrug zwischen 2 und 11 Jahren (Median = 4, vgl. Tab.1). Alle Probanden wurden zumindest partiell in der deutschen Sprache im Herkunftsland unterrichtet, bei den Aussiedlern wurde in der Regel ein basaler Gebrauch der deutschen Sprache in der Familie angegeben. Alle Personen bezeichneten die russische Sprache als Erst- (L1), die deutsche als Zweitsprache (L2), bei dem Probanden mit 11-jährigem Aufenthalt wurde zusätzlich unabhängig von der Reihenfolge des Erwerbs die Zweitsprache (deutsch) als aktuell vertrautere Sprache angegeben. Eine Untersuchung mittels der modifizierten Form des OldfieldHändigkeitsfragebogens [15] ergab für alle Personen eine eindeutige Zuordnung als Rechtshänder.

Tab. 1 Darstellung des Probandenkollektivs

\begin{tabular}{llllll}
\hline n & Kode & Geschlecht & Händigkeit & Alter & $\begin{array}{l}\text { Dauer des Zweit- } \\
\text { sprachgebrauchs } \\
\text { (in Jahren) }\end{array}$ \\
\hline 1 & RG & m & rechts & 29 & 2 \\
2 & RA & w & rechts & 19 & 3,5 \\
3 & RG & w & rechts & 27 & 4 \\
4 & RG & m & rechts & 31 & 4 \\
5 & RA & m & rechts & 32 & 4 \\
6 & RG & w & rechts & 26 & 5 \\
7 & RA & m & rechts & 21 & 11 \\
\hline
\end{tabular}

(RA = russlanddeutsche Aussiedler; $R G$ = russische Gastwissenschaftler, $\mathrm{m}=$ männlich, $\mathrm{w}=$ weiblich)

\section{Aufgabendesign}

Bei dem Wortflüssigkeitsparadigma (verbal-fluency) handelt es sich um eine Wortgenerierungsaufgabe, bei der die Probanden in einer vorgegebenen Zeit möglichst viele Substantive produzieren müssen. Die Aufgabe wird dabei entweder nach Vorgabe eines bestimmten Anfangsbuchstabens (phonematische Bedingung, z.B. - A: Apfel, Ampel, Arbeit ...), oder nach Vorgabe eines Oberbegriffs, zu dem die zu generierenden Wörter zugeordnet werden müssen (kategoriale Bedingung, z.B. - Tiere: Elefant, Tiger, Hund ...), durchgeführt. Die Probanden hatten vor Beginn der fMRT-Aktivierung die verbalen Instruktionen sowohl in russischer als auch in deutscher Sprache erhalten. Die Untersuchung wurde in zwei für die jeweilige Sprache separaten Durchgängen durchgeführt. Die Vorgabe des Buchstaben oder einer Kategorie erfolgte in alternierender Reihenfolge innerhalb des jeweiligen Durchgangs und in der entsprechenden Sprache. Als Baseline wurde die entspannte Ruhebedingung zwischen den Generierungsaufgaben registriert. Die Probanden sollten die während der Aktivierung zu generierenden Wörter leise vorsprechen (inner speech), ohne laut zu artikulieren, um artifizielle Kontaminierungen der fMRT-Aufzeichnung zu vermeiden. 


\section{fMRT-Untersuchung}

Die Datenakquisition erfolgte mit einem konventionellen Kernspintomographen (SIEMENS Magnetom Vision, Erlangen, 1,5 Tesla, $25 \mathrm{mT} / \mathrm{m}$ ) in Echo-Planar-Imaging (EPI)-Technik (TR $0,96 \mathrm{~ms}$, TE $59 \mathrm{~ms}$, FOV $220 \mathrm{~mm}$, Matrix $114 \times 128$, 16 Schichten, Ortsauflösung 1,45 mm*1,72 mm). Es wurden 80 Messungen mit 16 Schichten mit Angulation entlang der Temporallappenebene (axial) akquiriert. Den Aktivierungsphasen (phonematisches/semantisches Paradigma) folgten alternierend sog. Ruhephasen (jeweils 10 Messungen). Die Daten wurden auf eine separate Workstation (Ultra, Sun, Microsystems, CA, USA) transferiert und mittels SPM- (statistical parametric mapping) Softwaretools getrennt nach vier Kriterien jeweils für die Sprache und die Aufgabenbedingung versus Ruhezustand ausgewertet.

Die Daten wurden zunächst auf den ersten EPI-Datensatz bewegungskorrigiert angepasst (realigned). Die Parameter der Bewegungskorrektur wurden für alle drei Raumdimensionen über einen einfachen Least-Mean-Squares-Ansatz berechnet. Nach Durchführung einer t-Statistik mit Schwellenwertfestlegung wird mit Mehrfachtestung die statistische Relevanz überprüft. Die Art der Vergleiche (z. B. Aktivierung 1 mit Ruhe, Aktivierung 2 mit Ruhe etc.) wird hierbei über Kontraste festgelegt, das Ergebnis dieser Tests nachfolgend in Parameterdateien abgespeichert. Dabei ergänzen sich der Test auf Höhe der Aktivierung und jener auf Ausdehnung der aktivierten Region gegenseitig, wenn sowohl Regionen mit scharfer und hoher Aktivierung als auch solche mit größerer Ausdehnung aber geringeren z-Werten auftreten. Anschließend wurden die signifikant aktivierten Regionen (Cluster auf Aktivierungsebene) auf anatomische Bilder überlagert.

\section{Ergebnisse}

In Übereinstimmung mit vergleichbaren Untersuchungen bei Monolingualen [16,17] kam es bei der Untersuchung der Erstsprache zur Aktivierung eines breit angelegten Netzwerks verschiedener Strukturen, einschließlich präfrontaler, supplementär-motorischer, temporo-parietaler und zerebellärer Areale. Eine erhöhte Signalintensität des linken präfrontalen Kortex (LPFK) und des rechten Zerebellums zeigte sich konstant bei allen Probanden, während die Aktivierung anderer Areale mit hoher Intersubjekt-Variabilität einherging.

Eine Übersicht über die Hemisphären mit signifikanter Erhöhung der Signalintensitäten im präfrontalen Kortex wäh- rend der Wortflüssigkeitsaufgabe unter den entsprechenden Bedingungen findet sich in Tab. 2. Bei der Wortgenerierung in der Zweitsprache ergab sich hingegen eine auffällige Tendenz zu einer geänderten Verteilung der Aktivierungen. Die Aktivierung posteriorer Sprachareale zeigte kein konsistentes Beteiligungsmuster. Anterior kam es bei der überwiegenden Zahl der Probanden neben der nach wie vor ausgeprägten links-frontalen Aktivität zu einer deutlichen Mitaktivierung der rechten präfrontalen Region. Dieser Befund trat unter der kategorialen Bedingung am häufigsten auf. Bei einem Probanden (RA-5) zeigte sich für die kategoriale Bedingung in der Zweitsprache fast ausschließlich eine rechtsfrontale Aktivierung, während unter der phonematischen Bedingung überwiegend eine linksfrontale Beteiligung $\mathrm{zu}$ registrieren war. Die Analyse der Aktivitätsverteilungen desjenigen Probanden (RA-7), der in einem verhältnismäßig jungen Alter (im 10. Lebensjahr) nach Deutschland gekommen war und einen wesentlich längeren Gebrauch der Zweitsprache (11 Jahre) als die anderen Mitglieder der Probandengruppe (Median =4) aufwies, ließ in der Tat invertierte Verhältnisse in den Aktivierungsmustern deutlich werden. Bei der Ausführung der Wortflüssigkeitsaufgabe in der eigentlichen Erstsprache (russisch) kam es zu einer Mitaktivierung der rechten präfrontalen Region in der kategorialen Bedingung, während in seiner Zweitsprache (deutsch), die der Proband aktuell als die wesentlich vertrautere bezeichnete, ausschließlich die Aktivierung des linken präfrontalen Hirnareals zu registrieren war. Abb.1 zeigt neben einem charakteristischen Muster (RG-1) die ausgewählten Darstellungen der Aktivitäten für die genannten Beispiele (RA-5, RA-7) bezüglich aller Aufgabenbedingungen (Abb.1a-c).

\section{Diskussion}

\section{Die Rolle des präfrontalen Kortex bei der Sprachverarbeitung}

Die auch im Rahmen der vorliegenden Studie belegte Evidenz einer führenden Rolle der linken präfrontalen Region innerhalb des gewählten Paradigmas ist nicht überraschend. In der klinischen Forschung ist seit langem bekannt, dass nach frontalen Hirnläsionen deutliche Leistungsminderungen bei Wortflüssigkeitsaufgaben auch in Abwesenheit einer Sprachstörung auftreten. Die besondere Bedeutung des linken präfrontalen Kortex (LPFK) für mehrere, zur erfolgreichen Ausführung dieser Aufgabe notwendigen Funktionsschritte wie Initiation und Selektion, kognitive Flexibilität, verbales Arbeitsgedächtnis und Kategorisierung, wurde durch zahlreiche Läsionsstudien belegt [18]. Von Seiten der funktionellen

\begin{tabular}{|c|c|c|c|c|c|c|}
\hline$n$ & Kode & $\begin{array}{l}\text { Dauer des Zweit- } \\
\text { sprachgebrauchs } \\
\text { (in Jahren) }\end{array}$ & $\begin{array}{l}\text { L1 } \\
\text { phonematisch }\end{array}$ & kategorial & $\begin{array}{l}\text { L2 } \\
\text { phonematisch }\end{array}$ & kategorial \\
\hline 1 & RG & 2 & links & links & links & links/rechts \\
\hline 2 & RA & 3,5 & links & links & links/rechts & links \\
\hline 3 & RG & 4 & links & links & links & links/rechts \\
\hline 4 & RG & 4 & links & links & links & links/rechts \\
\hline 5 & RA & 4 & links & links & links & rechts \\
\hline 6 & RG & 5 & links & links & links & links \\
\hline 7 & RA & 11 & links & rechts/links & links & links \\
\hline
\end{tabular}

Tab. 2 Darstellung der Ergebnisse der fMRTAktivierung der präfrontalen Regionen nach Sprachen (L1 - Erstsprache, L2 - Zweitsprache) und Aufgabenbedingungen 


\section{Phonematisch}

L1
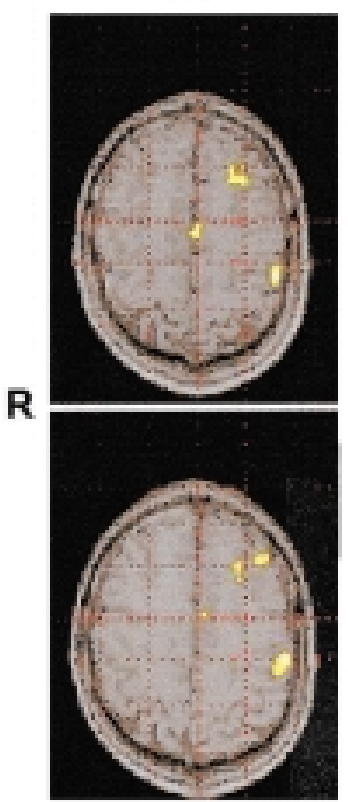

L2

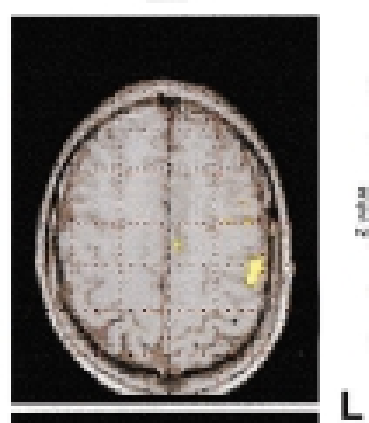

L

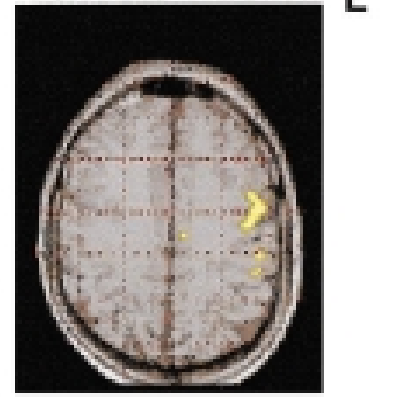

\section{Kategorial}

\section{L1}

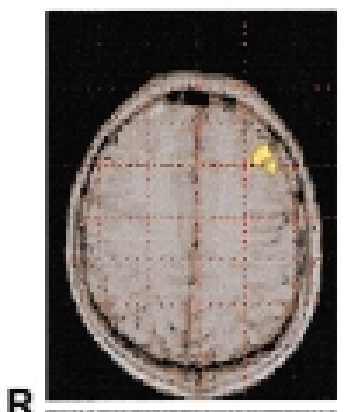

$\mathbf{R}$

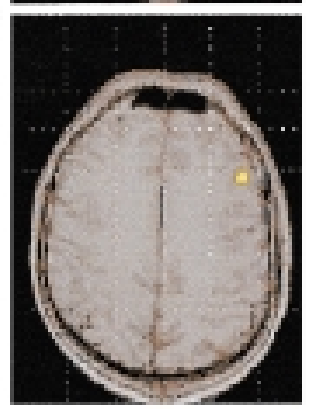

L2
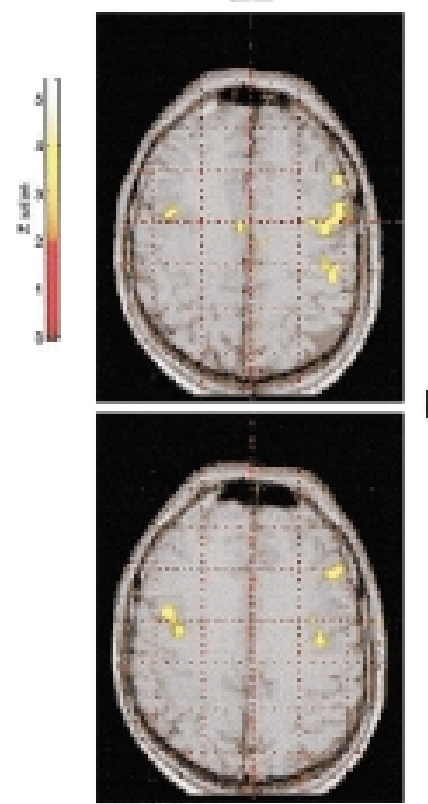

L

Abb. 1a Darstellung der fMRT-Aktivierungen eines repräsentativen Probanden (RG-1). In der kategorialen Bedingung der Zweitsprache zeigt sich eine zusätzliche Aktivierung der rechten präfrontalen Region.

Bildgebung haben inzwischen zahlreiche PET und fMRT Studien nach den ersten Evidenzen für eine besondere Bedeutung des LPFK für die semantische Wortanalyse [19] Hinweise auf eine Beteiligung dieser Struktur bei semantischen Aufgaben erbracht. Diese Beteiligung scheint unabhängig von der Modalität und dem Aufgabenmodus zu sein [20]. Sie betrifft

\section{Phonematisch}

L1
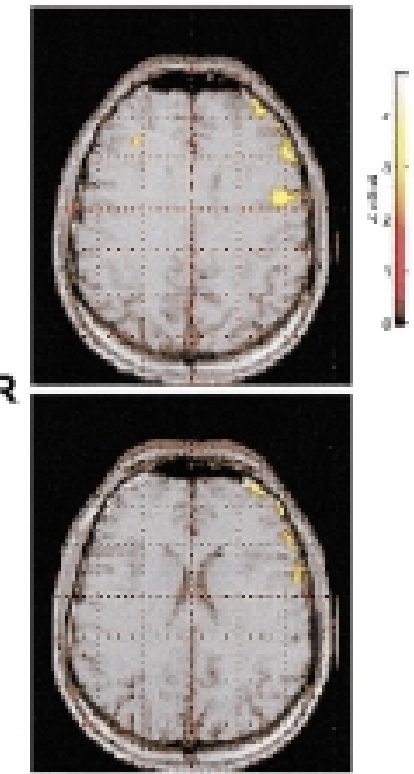

L2

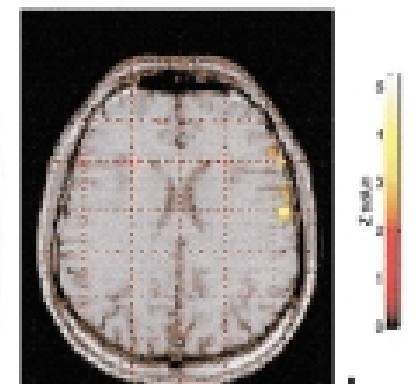

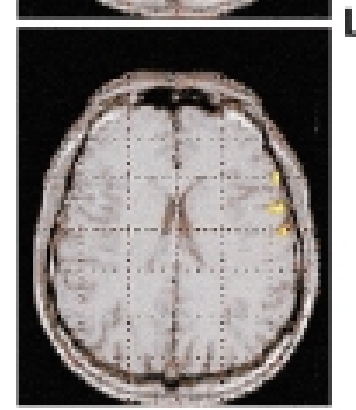

L

Abb. 1b Atypisches Aktivierungsmuster beim Probanden (RA-5) mit einem kompletten Wechsel nach rechts bei der kategorialen Bedingung der Zweitsprache.

sowohl Prozesse auf Wortbedeutungsebene (semantische Analyse), als auch auf der Wortformebene (lexikalische oder phonologische Analyse) [20,21] wobei sowohl die genauere aufgabenspezifische Verteilung innerhalb des LPFK für Analyse und Selektion als auch das Zusammenspiel mit den posterioren temporoparietalen Strukturen, die neben der 


\section{Phonematisch}

L1

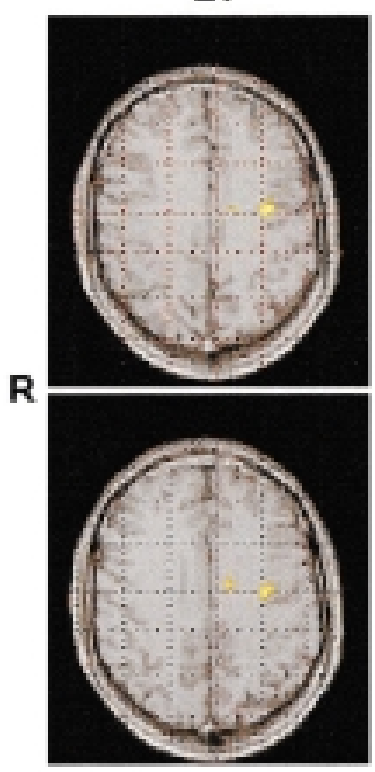

\section{L2}

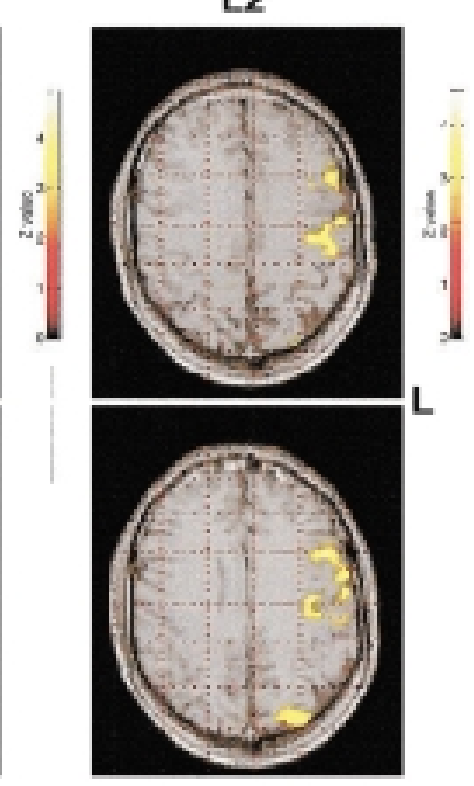

Kategorial

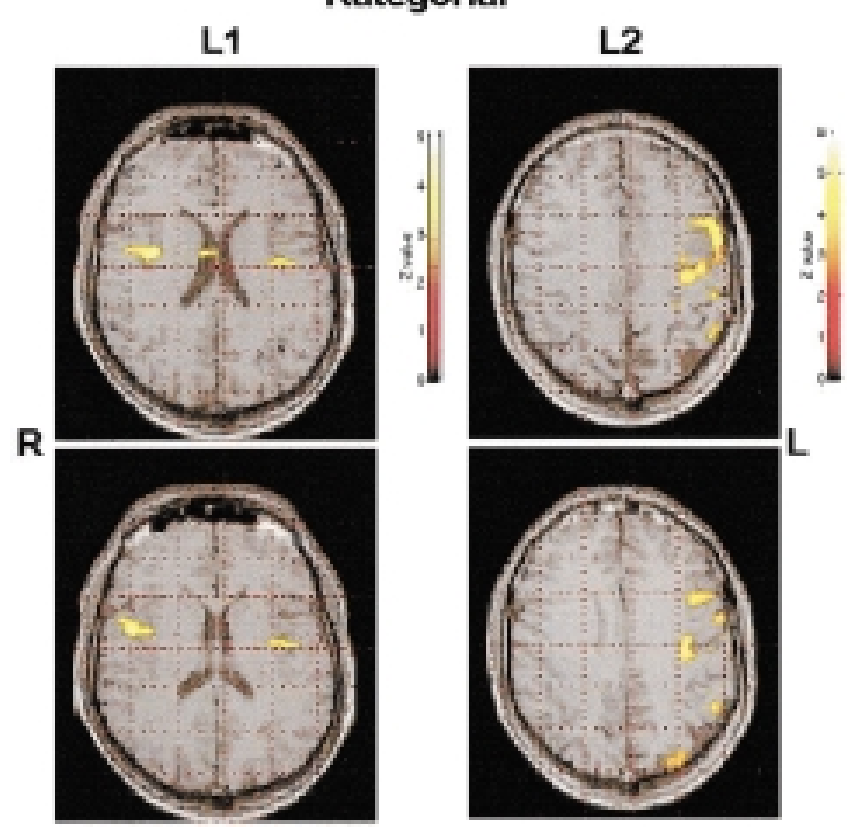

Abb. 1c Atypisches Aktivierungsmuster des Probanden (RA-7), der die längste Dauer des Zweitsprachgebrauchs aufweist. Hier zeigen sich rechthemisphärische Aktivierungen in der kategorialen Bedingung der Erstsprache.

akustischen Analyse den Puffer zu den abgespeicherten semantischen Informationen darstellen, aufgedeckt werden konnte [22]. Eine inkonsistente Beteiligung posteriorer Strukturen in unserem Experiment mag einerseits an dem gewählten „stummen“ Paradigma liegen, andererseits wird hiermit eine führende Rolle des LPFK beim Abruf von Informationen aus dem semantischen Gedächtnis belegt.

\section{Neuronale Korrelate domänenspezifischer Abrufstrategien}

In der gegenwärtigen Gedächtnisforschung wird von einer Unterteilung der Gedächtnisfunktion im Hinblick auf inhaltliche Aspekte der Informationsverarbeitung in verschiedene Komponenten ausgegangen [23], die für die Ebene der Enkodierung, der Abspeicherung bzw. des Abrufs jeweils unterschiedliche Beteiligungen neuronaler Strukturen aufweisen [24]. Es werden das episodische Gedächtnis (zeitlich und örtlich bezogene Einzelereignisse der persönlichen Vergangenheit), das semantische Gedächtnis (vom Kontext des Erwerbs abstrahierte Inhalte des allgemeinen Weltwissens), das prozedurale Gedächtnis (automatisiert abrufbare Fertigkeiten) und das Priming (ereigniskorrelierte Veränderungen im perzeptiven oder konzeptuellen Repräsentationssystem, die ohne bewussten Zugang die wiederholte Verarbeitung von Information erleichtern) unterschieden.

Der wesentliche Unterschied zwischen den ersten beiden als deklarativ geltenden Inhaltskomponenten des Gedächtnisses lässt sich folgendermaßen erklären. Die Erinnerung an Inhalte des episodischen Gedächtnisses erfordert in der Regel eine aktive, bewusste Rekonstruktion einer Reihe von Merkmalen, die mit dem Kontext der Erwerbssituation in einem engen Zusammenhang stehen wie z.B. dessen zeitliche und räumliche Charakteristika.

Beim semantischen Gedächtnis hingegen handelt es sich um ein Wissen i.S. einer immanenten Verfügung über eine Reihe von Inhalten wie Fakten oder Regeln, die beim Abruf keine aktive kontextuelle Rekonstruktion der Erwerbssituation verlangen. Traditionsgemäß wird angenommen, dass für diese beiden Gedächtniskomponenten bei Abspeicherung und Abruf Strukturen des limbischen Systems und des Frontalhirns von Bedeutung sind. Das prozedurale Gedächtnis und Priming gelten dagegen als implizit und greifen auf domänenspezifische kortikale bzw. subkortikale Areale zurück. Wie bereits in der Einführung erwähnt, erfordert das von uns gewählte Paradigma einen Abruf aus dem semantischen Gedächtnis. Aus den Ergebnissen der funktionellen Bildgebungsforschung lassen sich insbesondere über die Bedeutung der präfrontalen Regionen für den Abruf aus dem episodischen und semantischen Gedächtnis neue Erkenntnisse ableiten.

\section{Lateralisierte Abruf- und Enkodierungsfunktion}

Eine zusammenfassende Analyse diesbezüglicher Ergebnisse führte Tulving u. Mitarb. [25] zur Annahme eines so genannten HERA-Modells der Gedächtnisverarbeitung (Hemispheric Encoding/Retrieval Asymmetry). Nach den hypothetischen Vorstellungen dieses Konzepts sind die präfrontalen Regionen des Gehirns in asymmetrischer Weise an den unterschiedlichen Prozessen des Gedächtnisses beteiligt. Demnach soll der LPFK mehr in den Abruf aus dem semantischen Gedächtnis und in die parallele Enkodierung von neuen Informationen in das episodische Gedächtnis involviert sein als dies für den rechten präfrontalen Kortex (RPFK) zutrifft, während der RPFK mehr als der linke für den Abruf aus dem episodischen Gedächtnis zuständig sein soll. Bezogen auf die hier zur Diskussion stehende Befundlage könnte das Modell dergestalt zur Interpretation herangezogen werden, dass bei Bilingualen der Abruf von verbalen Informationen innerhalb der Zweitsprache in der Regel das episodische Gedächtnis zusätzlich 
beansprucht, während gleichartige Leistungen in der Erstsprache ausschließlich über das semantische Gedächtnis realisiert werden. Dies mag damit zusammenhängen, dass der Zugang zu den hochüberlernten Inhalten des semantischen Gedächtnisses der Erstsprache alleine nur über die semantische Abrufroute erfolgt, während die vergleichbare Beanspruchung des Gedächtnisses in der Zweitsprache nicht auf einen ähnlichen Grad der „Semantisierung“ zurückgreifen kann und deswegen kontextual bezogene Abrufstrategien aktiviert werden müssen, wie sie über das episodische Gedächtnis möglich sind. Denkbar ist ferner, dass solche Strategien gerade bei der kategorialen Bedingung des Wortabrufs eine wesentlich bedeutendere Rolle spielen können als bei der wortformbezogenen Strategie (phonematische Bedingung), was die Ergebnisse unserer Studie ebenfalls nahe legen.

Um diese Annahme weiter zu untermauern, wurden alle untersuchten Probanden zu ihren Generierungsstrategien im Nachhinein befragt. Die meisten gaben tatsächlich an, dass innerhalb des muttersprachlichen Paradigmas die Wörter viel leichter abgerufen werden konnten, während man sich in der Zweitsprache öfters an bestimmte Situationen erinnerte, wobei die zu generierenden Begriffe mit Hilfe kontextueller Information erinnert und benannt werden konnten. Ein Beispiel dafür wäre, dass man sich zu einer Kategorie wie „Tiere“ an einen Zoobesuch erinnert, bzw. sich bei „Lebensmittel“ einen Supermarkt vorstellt, um die Ausführung der Aufgabe in der Zweitsprache zu erleichtern. Diese „episodische“ Strategie war eher bei der kategorialen Bedingung behilflich, während man für die phonematische Bedingung meistens eine Wortanfangsergänzungs-Strategie in beiden Sprachen angab.

Die Ergebnisse desjenigen Probanden (RA-7, Tab. 2), dessen Aktivierungsmuster invertierte Verhältnisse gegenüber den anderen Gruppenmitgliedern aufwies, bedürfen einer besonderen Betrachtung. Entsprechend der oben vorgeschlagenen Interpretationslogik benutzt dieser Proband bei der Zweitsprache ausschließlich die semantische Erinnerungsroute, während er bei der (inzwischen weniger vertrauten, russischen) Erstsprache auf die Kompensation durch die kontextbezogene Strategie und somit auf die episodische Erinnerungsroute zurückgreift. Tatsächlich berichtete dieser Proband über Abrufschwierigkeiten in der Erstsprache sowie über kontextbezogene Strategien bei der kategorialen Bedingung in dieser Sprache.

Eine weitere aus der Analyse der Daten abzuleitende Überlegung, die allerdings noch zusätzlicher experimenteller Überprüfung bedarf, wäre, dass mit zunehmender Dauer des aktiven Sprachgebrauchs die rechtshemisphärische Beteiligung möglicherweise abnimmt (RG-6). Eine einfache Erklärung dafür wäre die Zunahme des Automatisierungsgrades und somit die Abnahme einer bewussten, episodischen Komponenten beim Abruf mit entsprechender „Semantisierung“ und Verlagerung der Aktivierung nach links. Der Status des semantischen Gedächtnisses i.S. der Zuordnung zu expliziten oder impliziten Prozessen ist noch nicht eindeutig geklärt [26]. Selbiges würde auch das Lexikon als einen wichtigen Bestandteil dieses Gedächtnissystems betreffen. Paradis [8] unterscheidet implizite linguistische Kompetenzen wie Phonologie und Morphosyntax, die auf prozedurale
Gedächtnismechanismen basieren sollen, vom Lexikon, für das zumindest zum Teil das deklarative Gedächtnis verantwortlich gemacht wird. Hierbei wäre zu klären, auf welchem Wege sowohl der Erwerb als auch die spätere Verfügbarkeit des Lexikons realisiert werden. Die Enkodierung und Abspeicherung von neuen Informationen im episodischen Gedächtnis erfolgt unter Beteiligung des limbischen Systems. Hinsichtlich des Erwerbs des semantischen Gedächtnisses besteht noch keine Einigkeit über die Rolle der hippokampaldienzephalen Strukturen. Squire u. Mitarb. [27] ordnen sowohl das episodische als auch das semantische Gedächtnis dem deklarativen Bereich zu, wobei der Erwerb des Zweiten als Überlernen der notwendigerweise über das Erste erworbenen Teile der Information betrachtet und somit die unbedingte Beteiligung der limbischen Strukturen für beide Gedächtnissysteme postuliert wird. Tulving und Markowitsch [28] gehen von einer Möglichkeit der doppelten Dissoziation für beide Systeme sowohl bei Einspeicherung als auch beim Abruf aus, wobei das hippokampale System nicht als entscheidend für die Einträge in das semantische Gedächtnis betrachtet wird. Die letztere Hypothese wurde neuerdings durch ein Bericht über drei Amnesiefälle im Kindesalter unterstützt [29]. Trotz verbleibender erheblicher Defizite des episodischen Gedächtnisses haben diese Patienten eine annähernd normale allgemeine Intelligenz einschließlich Wortschatz entwickeln können. Eine weitere Bestätigung findet sich in einer Publikation über einen erfolgreichen Erwerb der Zweitsprache bei einer Patientin mit anterograder Amnesie [30]. Andererseits gibt es mehrere Hinweise auf Schwierigkeiten des Wortschatzerwerbs bei Amnestikern sowohl in der Nativ- als auch in der Zweitsprache [31].

In der kognitiven Neuropsychologie gibt es seit langem Hinweise darauf, dass eine tiefere semantische Verarbeitung im Rahmen von Wortassoziationsaufgaben zu einer besseren Erinnerungsleistung für diese Wörter führt, als dies bei der perzeptuellen Enkodierung der Fall ist [32]. Die semantische Wortanalyse ist daher mit amnestischen Prozessen eng verknüpft. Bei dieser Verknüpfung scheint der LPFK eine besondere Rolle zu spielen [20]. Es kann demnach angenommen werden, dass bestimmte Informationen direkt über das Kurzbzw. Arbeitsgedächtnis im Wissenssystem unter Mediierung des LPFK gespeichert und abgerufen werden können. Erhebliche Abrufdefizite i.S. etwa einer retrograden Amnesie scheinen, wie aus der Läsionsforschung deutlich wird, nur bei kombinierten Schädigungen der frontalen und ipsilateralen temporopolaren Strukturen aufzutreten, also nur dann, wenn auch die wichtigen Verbindungen zu den posterioren Strukturen unterbrochen werden [24,33-35].

\section{Ausblick}

Zusammenfassend ist festzuhalten, dass die Ergebnisse unserer Studie die in der Einführung erwähnte Auffassung Paradis' $[8,9]$ über die primär linksseitige Repräsentation mehrerer Sprachen bei Bilingualen unter Annahme einer Reihe metalinguistischer Faktoren, die unter bestimmten Bedingungen eine rechtshemisphärische Beteiligung erklären können, unterstützen. So konnten wir eine primär führende Rolle des linken präfrontalen Kortex für die Ausführung der Wortflüssigkeitsaufgaben sowohl in der Erst- als auch in der Zweitsprache belegen. Für die unter bestimmten Aufgabenbedingungen registrierte zusätzliche rechtshemisphärische 
Aktivierung, konnte die Beteiligung mnestischer Prozesse im Rahmen der Sprachgenerierung verantwortlich gemacht werden. Vergleichbare Studien von Klein u. Mitarb. [13,36] konnten bei unterschiedlichen Gruppen von Bilingualen eine ausschließlich linkshemisphärische präfrontale Aktivierung im Rahmen von Wortgenerierungsaufgaben feststellen. Hierbei haben die Autoren Synonym-, Reim- und Verbgenerierungsaufgaben verwendet. Der entscheidende Unterschied zwischen diesen Paradigmen und der Wortflüssigkeitsaufgabe besteht darin, dass es sich bei ersteren ausschließlich um so genannte Single-word-Aufgaben handelt, d.h. die Probanden haben auf ein jeweils dargebotenes Wort mit nur einem Wort $\mathrm{zu}$ antworten. Eine solche Bedingung lässt nicht die von uns vorgeschlagene Interpretation über die Beteiligung des episodischen Gedächtnisses zu, so dass man hier von ausschließlich semantischen Abrufprozessen ausgehen sollte, was das Ausbleiben der rechts präfrontalen Aktivierungen erklären mag. Ferner lässt sich bei der Wortflüssigkeit im Unterschied zu den Single-Word-Aufgaben eine wesentlich größere Bedeutung der exekutiven Komponente für die Ausführung der Aufgabe annehmen, was nun innerhalb der Zweitsprache zu unterschiedlichen Aktivierungsmustern führen kann. Wir hoffen, dass den in dieser Arbeit aufgeworfenen Fragen im Rahmen der weiteren Forschung zur Bilingualität besondere Aufmerksamkeit gewidmet wird. Dies ist nicht nur für die theoretische Modellbildung zum Verständnis der Funktionsweise unseres Gehirns von Bedeutung, sondern mit Rücksicht auf eine nicht unerhebliche Anzahl bilingualer Mitbürger hinsichtlich z. B. der präoperativen Sprachdiagnostik auch von klinischer Relevanz.

Schließlich sind derartige Forschungsansätze und Erkenntnisse auch für eine Reihe von praxisbezogenen Problemen beim Zweitspracherwerb und insbesondere in der Rehabilitation von amnestischen und aphasischen Patienten von großer Bedeutung.

\section{Literatur}

${ }^{1}$ Broca P. Remarques sur le siège de la faculté articulé, suivies d'une observation d'aphemie. Bulletin de la Societé d'Anatomie (Paris) 1861; 2: 330-357

2 Wernicke C. Der aphasische Symptomenkomplex, eine psychologische Studie auf anatomischer Basis. Breslau: Cohn \& Weigert, 1874

${ }^{3}$ Pitres A. Etude sur l'aphasie chez les polyglottes. Rev Med 1885; 15: $873-899$

${ }^{4}$ Albert ML, Obler LK. The bilingual brain. New York: Academic Press, 1978

${ }^{5}$ Vaid J, Genesee F. Neuropsychological approaches to bilingualism: A critical review. Can Psychol Rev 1980; 34: 417-445

${ }^{6}$ Gordon HW. Cerebral organisation in bilinguals. Brain Lang 1980; 9: 255-268

${ }^{7}$ Hawellek B. Sprachorganisation im Gehirn bei mehrsprachigen Personen. Hamburg: Kovac, 1993

${ }^{8}$ Paradis M. Neurolinguistic aspects of implicit and explicit memory: implications for bilingualism and SLA. In: Ellis NC (ed). Implicit and explicit learning of languages. London: Academic press Harcourt Brace \& Company, 1995: 393-420

${ }^{9}$ Paradis M. The cognitive neuropsychology of bilingualism. In: deGroot AMB, Kroll JF (eds). Tutorials in bilingualism. New Jersey: Lawrence Erlbaum, 1997: $331-354$

${ }^{10}$ Hamers JF, Blanc MHA. Bilinguality and bilingualism. Cambridge: Cambridge university press, 1988
11 Obler LK, Hannigan S. Neurolinguistics of second language acquisition and use. In: Ritchie WC, Bhatia TK (eds). Handbook of second language acquisition. San Diego: Academic Press, 1996: 509-523

${ }^{12}$ Kim KHS, Relkin NR, Lee KM, Hirsch J. Distinct cortical areas associated with native and second languages. Nature 1997; 388 : $171-174$

${ }^{13}$ Klein D, Milner B, Zatorre RJ, Meyer E, Evans AC. The neural substrates underlying word generation: A bilingual functionalimaging study. Proc Natl Acad Sci USA 1995; 92: 2899-2903

${ }^{14}$ Dehaene S, Dupoux E, Mehler J, Cohen L, Paulesu E, Perani D, Van de Moortele PF, Lehericy S, LeBihan D. Anatomical variability in the cortical representation of first and second language. Neuroreport 1997; 8: 3809-3815

${ }^{15}$ Oldfield RC. The assessment and analysis of handedness: The Edinburgh Inventory. Neuropsychologia 1971; 9: 97-111

${ }^{16}$ Schlösser R, Hutchinson M, Joseffer S, Rusinek H, Saarimaki A, Stevenson J, Dewey SL, Brodie JD. Functional magnetic resonance imaging of human brain activity in a verbal fluency task. J Neurol Neurosurg Psychiat 1998; 64: $492-498$

${ }^{17}$ Ackermann H, Wildgruber D, Grodd W. Neuroradiologische Aktivierungsstudien zur zerebralen Organisation sprachlicher Leistungen. Fortschr Neurol Psychiat 1997; 65: 182 - 194

${ }^{18}$ Stuss DT, Benson DF. The frontal lobes. New York: Raven press, 1986

19 Petersen SE, Fox PT, Posner MI, Mintun M, Raichle ME. Positron emission tomographic studies of cortical anatomy of singleword processing. Nature 1988; 331: 585-589

${ }^{20}$ Gabrieli JDE, Poldrack RA, Desmond JE. The role of left prefrontal cortex in language and memory. Proc Natl Acad Sci USA 1998; 95: $906-913$

${ }^{21}$ Posner MI, Pavese A. Anatomy of word and sentence meaning. Proc Natl Acad Sci USA 1998; 95: 906-913

${ }^{22}$ Warburton E, Wise RJS, Price CJ, Weiller C, Hadar U, Ramsay S, Frackowiak SJ. Noun and verb retrieval by normal subjects: Studies with PET. Brain 1996; 119: 159-179

${ }^{23}$ Tulving E. Organisation of memory: Quo vadis. In: Gazzaniga MS (ed). The cognitive neurosciences. Cambridge: MIT Press, 1995: 839-847

${ }^{24}$ Markowitsch HJ. Which brain regions are critically involved in the retrieval of old episodic memory? Brain Res Rev 1995; 21 : $117-127$

${ }^{25}$ Tulving E, Kapur S, Craik FIM, Moscovitch M, Houle S. Hemispheric encoding/retrieval asymmetry in episodic memory: Positron emission tomography findings. Proc Natl Acad Sci USA 1994; 91: 2016-2020

${ }^{26}$ Markowitsch HJ. Memory and amnesia. In: Mesulam MM (ed). Principles of behavioral and cognitive neuropsychology. New York: Oxford University Press, 2000: 257-293

${ }^{27}$ Squire LR, Zola SM. Episodic memory, semantic memory, and amnesia. Hippocampus 1998; 8: 205-211

${ }^{28}$ Tulving E, Markowitsch HJ. Episodic and declarative memory: role of the hippocampus. Hippocampus 1998; 8: 198-204

${ }^{29}$ Varga-Khadem F, Gadian DG, Watkins KE, Connely A, Van Paesschen W, Mishkin M. Differential effects of early hippocampal pathology on episodic and semantic memory. Science 1997; 277: $376-380$

${ }^{30}$ Hirst W, Phelps EA, Johnson WK, Volpe B. Amnesia and second language learning. Brain Cogn 1988; 8: 105-116

${ }^{31}$ Ellis NC. Vocabulary acquisition: the implicit ins and outs of explicit cognitive mediation. In: Ellis NC (ed). Implicit and explicit learning of languages. London: Academic press Harcourt Brace \& Company, 1994: 211 - 282

${ }^{32}$ Craik FIM, Lockhart RS. Levels of processing: A framework for memory research. J Verb Learn Verb Beh 1972; 11: 671 - 684 
33 Markowitsch HJ, Calabrese P, Neufeld H, Gehlen W, Durwen HF. Retrograde amnesia for world knowledge and preserved memory for autobiographic events. Cortex 1999; 35: 243-252

${ }^{34}$ Calabrese P, Markowitsch HJ, Durwen HF, Widlitzek H, Haupts M, Holinka B, Gehlen W. Right temporofrontal cortex as critical locus for the ecphory of old episodic memories. J Neurol Neurosurg Psychiat 1996; 61: $304-310$

${ }^{35}$ Markowitsch HJ, Calabrese P, Liess J, Haupts M, Durwen HF, Gehlen W. Retrograde amnesia after traumatic injury of the fronto-temporal cortex. J Neurol Neurosurg Psychiat 1993; 56: 988-992

${ }^{36}$ Klein D, Milner B, Zatorre R, Zhao V, Nikelski J. Cerebral organization in bilinguals: A PET study of Chinese-English verb generation. Neuroreport 1999; 10: 2841 -2846

\section{Dr. Dipl.-Psych. Pasquale Calabrese}

Neurologische Universitätsklinik

Bereich Neuropsychologie und neurologische Ambulanz

(Knappschaftskrankenhaus)

In der Schornau 23-25

44892 Bochum

E-mail: pasquale.calabrese@ruhr-uni-bochum.de

\section{BUCHBESPRECHUNG}

H. Helmchen, F. Henn, H. Lauter, N. Sartorius (Hrsg.): Psychiatrie der Gegenwart. Band 3. Psychiatrie spezieller Lebenssituationen. 4. Aufl. 2000. 8 Abb., 23 Tab., 645 S. Geb.

Berlin-Heidelberg: Springer-Verlag. DM 333,-; öS 2.431,-; sFr 300,-. ISBN 3-540-65 800-9

Auch in dem dritten Band der 4.Auflage der „Psychatrie der Gegenwart“ setzt sich die Tendenz zur "Globalisierung“ der Psychiatrie in Form der Internationalisierung der Autorenschaft fort. Der Band ist übersichtlich gegliedert in „Grundlagen der Entwicklung“, „Psychiatrie des Kindes- und Jugendalters“, „Psychiatrie des höheren Lebensalters“, in eine Abhandlung über soziokulturelle Kontexte bei psychischen Störungen, „Kritische Ereignisse“ und „Besondere Lebensbedingungen“ (Psychiatrie der Obdachlosigkeit, psychiatrische Aspekte geistiger Behinderung). U. M. Staudinger und P. B. Baltes beantworten ihre zu Beginn ihres Artikels „Entwicklungspsychologie der Lebensspanne“ gestellte Frage, ob sich der Mensch ein Leben lang entwickelt oder ob die Entwicklung mit dem Erreichen des frühen Erwachsenenalters aufhört, eindeutig mit der Beschreibung der lebenslangen Entwicklung als dynamisches System. Die Vorteile der evolutionären Selektion würden im Lebensverlauf mit höherem Alter geringer, mit dem Lebensalter steige der Bedarf an Kultur, deren Wirkungskraft im Alter abnehme. Für die praktische Psychiatrie wichtig ist die Unterscheidung von Mechanik und Pragmatik der Intelligenz. Die Einbußen der Hochaltrigen (nach dem 80. Lebensjahr) werden nicht verschwiegen. H. Remschmidt und F. Fombonne weisen in ihrem Beitrag zur Entwicklungspsychopathologie darauf hin, dass diese erst vor kurzem zu einem interdisziplinären und integrativen Forschungsgebiet geworden ist. Das vorherrschende Merkmal der Analogieschlüsse zu Erwachsenen wird für die Kinder- und Jugendpsychiatrie abgelehnt. In der Entwicklungspsychopathologie müssten biologische, psychologische und psychosoziale Dimensionen der Entwicklung zu einer integrativen Sichtweise zusammengefasst werden. H. Remschmidt und M. H. Schmidt geben in ihrem Beitrag „Kinder- und Jugendpsychiatrie und -psychotherapie als klinische und wissenschaftliche Disziplin: Eine Einführung“" einen kurzen Aufriss der Entwicklung des Faches, der Versorgung, der Fachgesellschaften, der Aus-, Weiter- und Fortbildung, ethischer Probleme und der Zukunftsperspektiven. Die Tatsache, dass die kinder- und jugendpsychiatrische Forschung in den letzten Jahren eine gewisse methodische Eigenständigkeit entwickelt habe, wird betont. Als besonders bedeutende Entwicklungstendenzen werden eine stärkere Hinwendung zu den biologischen Grundlagen seelischer Erkrankungen und eine stärkere Berücksichtigung des Entwicklungsgedankens festgestellt. Diagnostik und Klassifikation in der Kinder- und Jugendpsychiatrie werden von denselben Autoren relativ kurz abgehandelt. Ebenfalls von Remschmidt und Schmidt wurden die Beiträge „Epidemiologie und Pathogenese in der Kinder- und Jugendpsychiatrie" und „Therapie in der Kinder- und Jugendpsychiatrie“ geschrieben. Die psychoanalytische bzw. psychodynamische Behandlung i.S.des verbalen Vorgehens ist bereits im Jugendalter möglich. Die kognitive Verhaltenstherapie ist bereits im Kindesalter nach Auffassung der Autoren anwendbar. Großer Wert wird auf die gruppenzentrierten Behandlungsmethoden gelegt. In der Aufzählung der Neuroleptika, die bei psychotischen Erkrankungen im Kindes- und Jugendalter empfohlen werden, sind, bis auf Clozapin, die atypischen Neuroleptika nicht enthalten. In der Beurteilung der Antidepressiva spielt das Kielholz-Schema von 1971 noch eine Rolle. Die Antidepressiva der jüngsten Generation (SSRI) werden im einzelnen nicht berücksichtigt. Den umfangreichsten Teil des Abschnitts „Psychiatrie des Kindes- und Jugendalters“ bildet die Darstellung der Störungsbilder (ebenfalls von H. Remschmidt und M. H. Schmidt). Die komprimierte, dabei alles Wesentliche enthal- 
tende Darstellung der Verfasser lässt ihre große klinische Erfahrung erkennen. Verdienstlich ist auch die Beschreibung von erwachsenentypischen Störungen, die bereits gegen Ende des Kindesalters und in der frühen Adoleszenz auftreten. Die traditionelle Einteilung der Schizophrenien in paranoide Schizophrenie, Hebephrenie, katatone Schizophrenie und Schizophrenia simplex wird durchaus beibehalten, im übrigen beruht die Klassifikation auf der ICD-10. In ihrem Kapitel „Diagnostische Probleme in der Psychiatrie des höheren Lebensalters“" gehen $\mathrm{H}$. Helmchen und $\mathrm{H}$. Lauter auf die Schwierigkeit ein, die Abgrenzung von den psychischen Veränderungen des nicht pathologischen Alterns, die Abgrenzung von Beschwerden und Symptomen körperlicher Erkrankung und Behinderung und die Abgrenzung psychischer Störungen untereinander vorzunehmen. Es wird erkennbar, dass die gestiegene Lebenserwartung und größere Gesundheit zu einer Differenzierung der dritten Lebensphase jenseits des 70. Lebensjahres in ein durch aktiven Lebensstil charakterisiertes „drittes Lebensalter" bis etwa zum 85. Lebensjahr und jenseits davon in ein „viertes Lebensalter" mit zunehmender körperlicher Gebrechlichkeit geführt haben. Die Relativierung negativer bzw. positiver Altersstereotype wird ausdrücklich nahegelegt. Die Bedeutung klinischer Kriterien zur Erkennung von depressiven Störungen bei kognitiven Beeinträchtigungen wird im Zusammenhang mit der Diskussion um die „depressive Pseudodemenz“ unterstrichen. R. Ferszt und S. Kanowski führen aus, dass sich die intuitiv erwartete Beruhigung und nachlassende Dynamik bei vielen Krankheitsbildern im Alter aufgrund neuerer Studien bestätigen lassen. Die tradierte Vorstellung einer generell schlechten Prognose der Schizophrenien sei sicherlich nicht aufrechtzuerhalten. Das epidemiologisch wichtige Kapitel „Depression im höheren Lebensalter“ haben M. Blanchard und N. Graham verfasst. Die in der Literatur häufige Feststellung, nach der die Suizidraten bei älteren Menschen am höchsten sind, wird bestätigt. Die Elektrokrampftherapie wird für die wenigen älteren Menschen mit schwerer, auf andere Behandlungsmethoden nicht ansprechbarer Depression als eine nach wie vor wichtige Behandlungsalternative bezeichnet. Der Freud'schen Ablehnung der Psychotherapie im höheren Alter wird widersprochen. Die Kapitel „Psychiatrische Behandlung und Rehabilitation älterer seelisch Kranker“ (J. Wertheimer) und „Psychiatrische Aspekte des Lebensendes“ (Th. Fuchs, H. Lauter) beschließen die „Psychiatrie des höheren Lebensalters“. Auch Fuchs und Lauter bestätigen, dass im allgemeinen Todeswünsche oder Suizidgedanken alter und höchstaltriger Menschen selten sind. Von besonderem Interesse für den Psychiater ist die Erörterung der „Nahtodeserlebnisse“. Unter dem Rubrum „Soziokultureller Kontext“ werden psychiatrische Erkrankungen bei Frauen (I. F. Brockington und M. Lanczik), kulturspezifische psychische Störungen (W. G. Jilek, L. Jilek-Aall) und die Psychiatrie in Entwicklungsländern (N. Sartorius) behandelt. Sartorius macht auf die Unbegründetheit einer einfachen Einteilung der Welt in Entwicklungsländer und entwickelte Länder anhand von Beispielen aufmerksam. Der Mythos von der unterschiedlichen Häufigkeit psychischer Störungen zwischen den entwickelten und den Entwicklungsländern habe an Kraft verloren. Es gebe kulturspezifische Ausprägungen psychischer Erkrankungen, die größten Unterschiede zwischen der entwickelten und der sich entwickelnden Welt resultierten aus der Armut. Die Internationalisierung der Autorschaft erscheint fraglos sinnvoll in dem Abschnitt „Kritische Ereignisse“, in dem psychiatrische Probleme aufgrund extremer Umweltbedingungen (V. Krasnov, V. Lebedev und E. West), psychiatrische Probleme infolge von Naturkatastrophen und von Menschen ausgelösten Katastrophen (E. J. Bromet), psychiatrische Probleme im Zusammenhang mit Verfolgung und Flüchtlingsstatus (J. de Jong), psychiatrische Probleme im Zusammenhang mit Folter (L. Tata Arcel, I. Genefke, M. Kastrup), psychiatrische Probleme im Zusammenhang mit Gewalt und Vergewaltigung (V. Folnegović-Šmalc) und Psychiatrie in Haft, Gefangenschaft und Gefängnis (N. Konrad) erörtert werden. Krasnov et al. definieren das neue Fachgebiet der ökologischen Psychiatrie als einen Bereich komplexer wissenschaftlicher Erforschung der seelischen Gesundheit und Krankheit im Bereich anthropogen hervorgebrachter oder natürlich auftretender ungewöhnlicher Umweltbedingungen. Sie beschreiben das Syndrom der sog. Liquidatoren der Folgen der Katastrophe von Tschernobyl (Asthenie, somatoforme Symptome, Dysthymie, kognitive Dysfunktion, vegetative Dysregulationen, psycho-somatische Störungen). Bromet bedauert den Mangel an Katastrophenuntersuchungen mit Längsschnittdesign oder ausgedehnten langfristigen Nachuntersuchungen. De Jong weist mit Recht auf die Wichtigkeit der posttraumatischen Belastungsstörungen (DSM-III) als Zusammenfassung verschiedener Syndrome hin. Zahlreiche Beobachtungen in der klinischen Praxis hätten die Vermutung gestützt, dass einige der Auswirkungen der Verfolgung von den Eltern auf die Kinder übertragen worden seien. Bei Untersuchungen an Kindern von amerikanischen Kriegsgefangenen des Vietnam-Krieges, bei Kindern von Holocaust-Überlebenden und bei Kindern, deren Eltern im Zweiten Weltkrieg in japanischer Lagerhaft gewesen seien, fanden sich Hinweise auf generationsüberschreitende Folgen der Gefangenschaft. Nach Konrad ist bei Langzeithaft eher eine zunehmende psychische Stabilisierung des Häftlings festzustellen. Der innere Widerstand der Inhaftierten gegen die Haftsituation sinke stetig. Im Kapitel „Besondere Lebensbedingungen“ werden die „Psychiatrie der Obdachlosigkeit“ (M. M. Fichter) und „Psychiatrische Aspekte geistiger Behinderung“ vielleicht ein wenig künstlich zusammengefasst. Fichter unterstreicht, dass in den meisten westlichen Industrieländern seit den 70er Jahren mit der Verteuerung des Wohnraumes und mit der Politik der Reduzierung psychiatrischer Betten die Obdachlosenproblematik sich verschärfte. In Nordamerika und in Nordeuropa wurden in den 80er und 90er Jahren kommunalpolitische Maßnahmen eingeleitet, die die Betroffenen durch Zurverfügungstellung von Wohnraum in Heimen und Pensionen von der Straße holten. Es bestehe derzeit allerdings immer noch ein erheblicher Mangel hinsichtlich der psychiatrischen und sonstigen medizinischen Versorgung dieser Personengruppe, die eine Lebenszeitprävalenz für schwere psychische Erkrankungen (Schizophrenie, typische affektive Erkrankung) von 15-30\% und für Alkohol- und Drogenmissbrauch bzw. -abhängigkeit von 60-70\% aufweist. Holland stellt fest, dass es für geistig behinderte Menschen im 20. Jahrhundert in nahezu sämtlichen Lebensbereichen dramatische Veränderungen gegeben habe. $\mathrm{Zu}$ den bemerkenswertesten gehöre, wie sich die Einstellung der Gesellschaft mit einer Abkehr von der Ideologie der Ausgrenzung und einer Zuwendung zu integrativen Bemühungen gewandelt habe. Der IQ wird als objektiver, standardisierter und reliabler Parameter zur Beurteilung der Fähigkeiten einer Person dargestellt, kognitive Aufgaben zu bewältigen. Auf kontroverse Ansichten zu dieser Frage wird hingewiesen. Die Probleme des sozialen Versorgungsbedarfs und der Förderung von Normalität erfahren eine angemessene Würdigung.

Band 3 der 4. Auflage der „Psychiatrie der Gegenwart“ ist in besonderer Weise geeignet, den fachlichen Bildungshorizont des Psychiaters durch die umgreifende Schilderung mancher nicht im Zentrum der fachspezifischen Aufmerksamkeit liegender Sachverhalte und Probleme zu erweitern. Auch der Erwachsenenpsychiater sollte sich hinsichtlich der Psychiatrie des Kindes- und Jugendalters kundig machen. Die Psychiatrie des höheren Lebensalters ist inzwischen ein Kernthema geworden, was für die unter der Überschrift „Kritische Ereignisse“ bzw. „Besondere Lebensbedingungen“ dargestellten Gegebenheiten nicht ohne weiteres gilt. Der Band stellt eine erwünschte Möglichkeit der Abrundung psychiatrischer Kenntnisse zur Verfügung und es ist zu hoffen, dass davon reichlich Gebrauch gemacht wird.

K. Heinrich, Düsseldorf 